\title{
COMPETITIVIDAD Y CALIDAD DE VIDA EN DESTINOS TURÍSTICOS DE MÉXICO
}

\section{COMPETITIVENESS AND QUALITY OF LIFE IN TOURIST DESTINATIONS IN MEXICO}

\author{
Pablo Sandoval Cabrera ${ }^{1}$
}

${ }^{1}$ Mexicano. Universidad de Guadalajara. Núcleo Universitario de los Belenes, Zapopan Jalisco, México. Email: pasaca2636@gmail.com

\begin{tabular}{|l|l} 
Recibido: 29.11 .18 & Aprobado: 25.05.19
\end{tabular}

DOI:10.15517/isucr.v20i41.38818

\section{Resumen:}

El presente ensayo tiene como objetivo central encontrar evidencias empíricas que nos permitan constatar la relación existente entre competitividad de las ciudades y áreas urbanas, medida a través del índice que elabora el Centro de Investigación y Docencia Económica; y la calidad de vida de las personas, utilizando un conjunto de indicadores de bienestar (pobreza, desarrollo humano, empleo, educación, salud, ingresos, entre otros).

Para cumplir con tal propósito, se realiza un análisis correlacional y comparativo entre competitividad y algunos indicadores, así como entre indicadores de bienestar en una muestra amplia de municipios turísticos de México en una primera etapa y entre una muestra restringida de 5 municipios que ofrecen servicios de turismo de playa.

La hipótesis que se plantea es que existe una relación positiva entre competitividad del destino y calidad de vida. Si bien los resultados no son tan contundentes, pareciera haber evidencias de que validad la hipótesis. Sin embargo, queda en claro la necesidad de realizar estudios de mayor profundidad y amplitud para darle una mayor robustez empírica al estudio. Palabras claves: competitividad; sustentabilidad; calidad de vida; destinos turísticos; bienestar;

\begin{abstract}
:
The main objective of this essay is to find empirical evidence that allows us to verify the relationship between the competitiveness of cities and urban areas, measured through the index prepared by the Center for Research and Economic Teaching; and the quality of life of people, using a set of indicators of well-being (poverty, human development, employment, education, health, income, among others). To fulfill this purpose, a correlational and comparative analysis is performed between competitiveness and some indicators, as well as between indicators of well-being in a large sample of tourist municipalities of Mexico in a first stage and between a restricted sample of 5 municipalities that offer services of beach tourism.
\end{abstract}

Key-Words: competitiveness; sustainability; quality of life; tourist destinations; well-being; 


\section{Introducción}

Tanto en el discurso académico como en político se menciona recurrentemente que el turismo es una alternativa, la mejor en algunos casos, para impulsar el crecimiento económico y mejorar la calidad de vida de comunidades que cuentan con atractivos naturales, culturales y/o arquitectónicos para ofrecer a sus visitantes. Regularmente se sobre dimensionan las "bondades" que ofrece con relación a la generación de inversión, empleo e ingresos, amén de los efectos multiplicadores que genera en el resto de la economía en cada una de los eslabones de la compleja cadena de valor que comprende el turismo.

Si bien es cierto, que muchas comunidades no disponen de la infraestructura, la estructura sectorial y, en algunos casos, las capacidades humanas para especializarse en otras actividades diferentes al turismo; como podría ser, la industria manufacturera, el comercio u otros servicios, también lo es el que en el caso de México la aparición de la mayoría de destinos turísticos ocurrió debido al impulso de políticas públicas exprofeso, siendo el caso más conocido el de los llamados Centros Turísticos Integralmente Planeados (CIP), entre los que se cuentan Cancún, Loreto y Los Cabos, entre otros.

Una pregunta que se debe plantear al menos, es si el turismo fue la mejor, sino es que la única, alternativa de crecimiento económico para aquellos municipios del país que son esencialmente turísticos, una muestra de los cuáles se analiza en este estudio.

De igual manera conviene preguntarnos si existe al menos una notable correlación entre turismo y calidad de vida lo suficientemente robusta como para validar lo que se pregona en la academia y la política, como lo anotamos líneas arriba.

En el mismo sentido partiendo de una concepción de competitividad que intenta superar la disyuntiva teórica Porter versus Krugman que en esencia se confrontan al reconocer, el primero de los autores, que son las empresas en el marco de un complejo entramado de interrelaciones con elementos (competidores, complementadores, ambiente natural, instituciones, políticas públicas, etc) del contexto en el que se ubican; en tanto el segundo de ellos insiste en que la competitividad se reduce a la productividad y, por tanto, es una tarea exclusiva de las firmas (es un tema de costos y eficiencia) y en todo caso el locus sería relevante en todo caso al aportar alguna ventaja de localización o ubicación geográfica de las empresas.

En este ensayo se busca abonar el dirección a una nueva conceptualizacón de competitividad donde la misma se concibe en su complejidad y multidimensionalidad, entendida no como un fin en sí mismo, sino como un medio para alcanzar mayores niveles de bienestar y calidad de vida de quienes habitan los territorios, que son desde nuestra perspectiva, quienes finalmente compiten. Es decir la competitividad se convierte en esta nueva concepción, en una tarea social que es compromiso de múltiples sujetos (empresas, gobiernos, comunidades) y que entendida como un medio adquiere sentido al contribuir a alcanzar más altos estándares de calidad de vida. 
La calidad de vida debe ser entendida no solamente en términos de acceso sino también de inclusión. El ejemplo más ilustrativo para clarificar este nuevo significado de calidad de vida tiene que ver con el trato diferenciado hacía las mujeres existente, aún hoy, en ámbitos laborales diversos. Si bien es que las mujeres ya pueden laborar, prácticamente en cualquier actividad productiva (acceso), también lo es el hecho de que reciben menores ingresos que los hombres, a trabajos iguales (inclusión), sólo por el hecho de ser mujeres. Este fenómeno se reproduce en diversos ámbitos de la vida social, tales como la educación, la salud y la política y lo sufren también otros grupos sociales por razones, étnicas, etáreas, religiosas y/o culturales.

De la muestra de municipios turísticos de México analizados en este ensayo se busca evidenciar los impactos que ha tenido el turismo en la calidad de vida, al mismo tiempo que se intenta dilucidar si se relacionan con los niveles de competitividad observados en dichos destinos. De acuerdo al análisis realizado, las evidencias parecieran no ser tan contundentes especialmente para el caso de los destinos de turismo masivo, como Mazatlán, Cancún, Puerto Vallarta, Los Cabos y Acapulco.

\section{Importancia del turismo}

El turismo es, sin duda, una de las actividades económicas más dinámicas y de mayores impactos en muchas regiones y comunidades del mundo. Los estudios que se han realizado para evaluar sus impactos en términos de crecimiento económico, arrojan resultados positivos. Esto es, hay una relación directa y positiva entre una mayor dinámica del turismo y un mayor crecimiento económico.

La Secretaria de Turismo (2000) estimó, en su estudio de gran visión del turismo en México, que para el año 2020 los ingresos por turismo en el mundo, se acercarían a la cifra de 600 mil millones de dólares, estimación que seguramente se vio afectada por la reciente crisis de los años 2008-2009.

De cualquier manera, el crecimiento del sector es muy importante y su contribución a las economías nacionales y locales no es nada despreciable. De acuerdo a datos oficiales (SECTUR, 2010) la contribución del turismo a la generación de riqueza nacional, medida por el Producto Interno Bruto (PIB), ronda el 8\% y en algunos Estados y municipios del país su contribución es mayor.

Para una gran cantidad de economías estatales el turismo representa una importante derrama económica. Empezando por Quintana Roo en donde representa el 48\%, Baja California Sur, cerca del 30\%; Nayarit, Guerrero y Sinaloa entre el 13 y 17 por ciento. En tanto para cerca de 10 estados, entre los que se encuentra Jalisco, significa entre el 5 y 10 por ciento en la generación de riqueza

Para el caso de Jalisco, considerando solamente el sector de servicios de alojamiento y preparación de alimentos, aportó un valor de 24,100 millones de pesos en el año 2010 (INEGI, 2011), representando el 3.1\% del valor total generado por la economía en ese año. Para el año 2012, una vez superada la crisis de 2007-2009 -por la recesión económica sufrida, 
así como por la contingencia ambiental- se estima que la aportación del turismo al PIB estatal fue cercana al 5\% con un valor superior a los 30 mil millones de pesos corrientes.

Previo a la crisis, el turismo representaba cerca de 2.5 millones de empleos directos en el país (INEGI-SECTUR, Cuenta satélite del turismo), aproximadamente el 6\% de los empleos totales del país. Adicionalmente se estima que genera alrededor de 3 empleos indirectos por cada empleo directo, hecho que da cuenta de su importancia en términos de encadenamientos con una gran cantidad de ramas y actividades productivas.

El turismo sobresale también en términos de productividad. De acuerdo a datos de Datatur e Inegi (2009) la productividad promedio anual por trabajador era de 370 mil pesos, en tanto la media de la economía fue de 265 mil pesos, lo que significa que un empleado de este sector es aproximadamente $40 \%$ más productivo con respecto al trabajador promedio del país. Sin embargo, comparativamente con un empleado del turismo en países como Estados Unidos o Francia, la productividad promedio de un empleado mexicano es apenas de un tercio, de la de trabajadores de estos países.

En términos de ingresos el turismo no escapa a las condiciones de precariedad salarial que imperan en la mayoría de empleos que se ofrecen en el país, aunque presenta condiciones relativamente mejores.

De acuerdo a un estudio realizado por el Colegio de México en el año 2011 titulado "Análisis económico del mercado laboral en el sector turístico: Hacia una política pública para la igualdad entre hombres y mujeres" los ingresos por hora para el año 2010 de empleados en actividades turísticas eran de 27.80 pesos para los hombres y de 21 pesos para mujeres, de tal suerte que el ingreso por jornada laboral de 8 horas un trabajador del sector turismo podía percibir un ingreso cercano a los 220 pesos por persona.

Comparativamente con empleados del mismo sector en otros países como Alemania o España, a pesar de lo deprimido de sus mercados laborales, las percepciones por hora son superiores a los 3 Euros, estableciendo como tope mínimo un rango de entre 1.70 y 2.00 euros por hora, para un empleado tipo del sector comercio y de servicios, de acuerdo al "Job center" de Alemania.

A nivel de la economía de los municipios turísticos, los impactos son aún mayores al constituirse en el principal motor de la economía, como ocurre con municipios como Cancún, Puerto Vallarta y Mazatlán, por citar sólo algunos.

Sus efectos positivos se manifiestan, además, al evitar la emigración de la población local, fomentar el intercambio cultural entre regiones y países y la mejora del nivel socioeconómico de la población local, entre otros (Sandoval, Mimeo).

Debido a su innegable importancia económica a todos los niveles, es necesario propiciar las condiciones que garanticen su sustentabilidad y competitividad futura en la perspectiva de afectar favorablemente las condiciones sociales y económicas de quienes viven del turismo. 


\section{En torno al concepto de competitividad}

La competitividad, al menos desde finales de los años ochenta, ha sido un tema recurrente en los círculos de los policy maker, gobiernos y empresarios, se ha convertido en tema central de la política y la economía desde entonces. Inevitablemente, ha transitado del ámbito de la política y la económica a la academia. A pesar de lo cual, continua siendo tres décadas después, un concepto difícil de definir. Budd y Hirmis (2010: 1016) mencionan que "el término competitividad es aplicado a una amplia variedad de negocios y circunstancias económicas. Consecuentemente significa diferentes cosas para diferentes personas"

Generalmente hacemos referencia "a la capacidad o tendencia a competir bajo condiciones de mercado. En particular, denota la habilidad para ganar y mantener una posición en mercados competitivos, hecho que se manifiesta en un mayor éxito de los negocios, participación de mercado y beneficios" (Turok, 1999, citado por Lengyel, 2004: 1).

El mismo Lengyel (2004: 3) señala que en el contexto de globalización económica dos asuntos adquieren especial relevancia:

1. El interés en alcanzar la mayor concentración de las actividades económicas como alternativa para lograr una ventaja competitiva (por las economías de escala que se generan) y se convierten en uno de los mayores atributos de la economía urbana y regional.

2. La mejora de la competitividad, como un asunto clave para las políticas económicas y regionales en la perspectiva de entender los cambios de la competencia global.

Ambos aspectos deben ser integrados en una noción estándar de competitividad, cuyo propósito principal debe ser aportar un marco de análisis para identificar, no sólo el éxito económico sino también los impactos que este tiene sobre el bienestar y calidad de vida.

Con el fenómeno de la globalización, intensificado por el desarrollo de las tecnologías de la información y la comunicación (TICs), se impone otro sentido a la competencia. La misma se constituye en "parte integral del movimiento global de acumulación del capital, "es por lo tanto, el motor básico de la dinámica capitalista" (Posas, 1985: citado por Muller, 1995: 138). La competitividad, desde la óptica de Muller (1995: 138) es un término en boga que hace referencia a la competencia entre capitales por apropiarse de los mejores espacios de valorización a escala global. En este sentido, competencia y competitividad tienen el mismo origen pero distinta escala. Lo que se observa es un fenómeno de acumulación a escala global (globalización en sentido estricto) dinamizado por las TICs que envuelve todas las esferas de la vida social, por lo que cobijado en esta realidad el mismo autor sostiene que no hay razón para debatir cuales son los componentes de la competitividad, está constituye un fenómeno global en un doble sentido: Primero por no reconocer fronteras de ningún tipo y, segundo por considerar todas las dimensiones de la vida social, incluyendo la relacionada con el medio ambiente. 


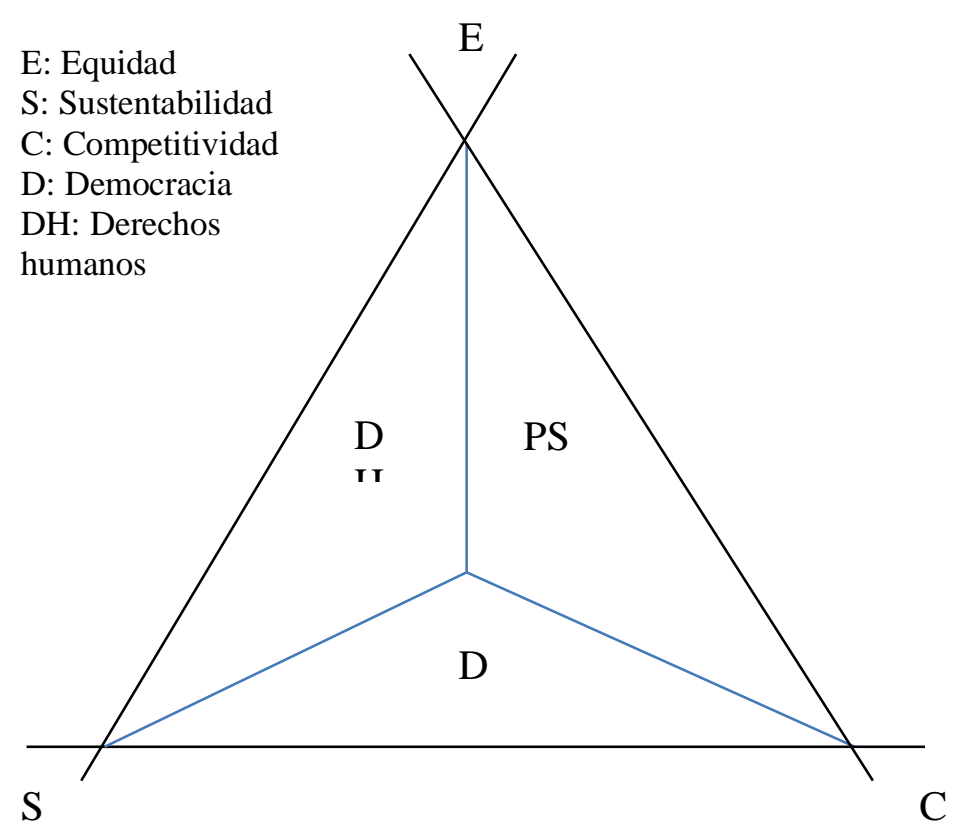

Figura 1: Relación de competitividad con otros conceptos y con valores sociales. Fuente: Muller, Geraldo: El caleidoscopio de la competitividad, Revista de la CEPAL. No.58, agosto de 1996.

La competitividad, entonces, estaría relacionada con el resto de componentes y valores que estructuran y definen la dinámica social, por tanto, con la noción de desarrollo, tal y como se ilustra en la figura de arriba. En la base de la pirámide estarían la sustentabilidad (S), la competitividad (C) y la democracia dando soporte a la equidad como fin primordial en un contexto institucional de participación social (PS) y respeto a los derechos humanos (DH). Entendida la competitividad como un fenómeno multidimensional, su inevitable relación con el desarrollo y, por tal con las posibilidades que tiene la gente de mejorar sus condiciones de vida, obligan a los estudiosos del tema a ampliar los análisis más allá de los factores estrictamente económicos que, sin duda, son importantes.

Sin embargo, es preciso reconocer que el debate sobre competitividad, sigue girando en gran medida entre la posición de Krugman (1990), que plantea que la competencia se da entre firmas fundamentalmente y la de Porter (1996) que sostiene que no se puede entender la competitividad de las empresas al margen del contexto en el que se ubican.

Desde su origen, el constructo competitividad se ha prestado al debate. En el contexto de la teoría económica se distinguen dos corrientes, cada una sustentada en lo particular en alguna de las posturas señaladas: una de ellas plantea que los territorios compiten en el mercado internacional, en tanto la otra, en afinidad con la noción krugmaniana, sostiene que son las empresas las que compiten. 
En un polémico trabajo Krugman (1994) se atrevió a señalar que se estaba abusando en el uso del concepto de competitividad, además de que su abordaje reflejaba una notable falta de rigor científico. Acusaba, que incluso muchos de los estudios empíricos que se habían realizado con el propósito de medir la competitividad tenían la intención de darle soporte a ideas preconcebidas más que fines explicativos. En un trabajo posterior irónicamente afirma que la "competitividad es para algunos, una forma poética de llamar a la productividad" (1996: 2). Enfatiza, que no existe diferencia significativa entre la competitividad de una empresa y una región o país, sólo son formas distintas de ver la competencia a escala global, siendo los países, finalmente, un conglomerado de empresas que compiten en el mercado mundial.

En torno a la visión de Krugman existen una gran cantidad de trabajos de investigación que intentan determinar cuáles son las condiciones, básicamente de carácter técnico económico (que inciden en eficiencia en costos), que definen las ventajas competitivas de las empresas, entre ellos podemos ubicar a los realizados por Siggel (2007), Sobrino (2005) y Body (1999), entre otros.

Pero también algunos que intentan refutar sus argumentos. Entre estos destaca el realizado por Camagni (2002) quien expresa firmemente, que puede demostrarse que la postura de Krugman es errónea, en la medida que se puede comprobar que:

a) Las empresas utilizan la localización como instrumento competitivo, y la movilización global para optimizar costos de producción y distribución;

b) Los territorios no son meros objetos pasivos de las decisiones de localización por parte de las empresas, sino que son comunidades constituidas por sujetos económicos que actúan en interés propio, intentando mantener o atraer empresas;

c) Las empresas se basan cada vez más en externalidades, bajo la forma de bienes públicos suministrados por la administración pública local; y

d) Las empresas locales necesitan cada vez más bienes externos seleccionados y recursos específicos, que pueden ser difíciles de conseguir rápidamente sobre la única base de los desarrollos espontáneos del mercado.

Para empezar hay una diferencia importante las ciudades, regiones o países y las empresas las primeras no pueden ir a la quiebra si no son competitivas, las empresas sí (Turok, 2004). Lo que es más, ni siquiera es determinante tener una participación proporcionalmente grande -con relación al tamaño de una economía- en el comercio internacional para ser competitivo. "En Estados Unidos sólo el 10\% de su producto interno bruto (PIB) se explica por exportaciones, el resto tiene un origen interno".(Yap, 2004: 3). De hecho, históricamente ha mantenido un déficit comercial sin mayor pérdida de competitividad. Por esta razón, entre otras, es muy complicado trasladar el concepto de competitividad del nivel micro al nivel macro.

Afortunadamente el análisis de la competitividad se ha ampliado y ha rebasado la visión estrictamente economicista hasta incursionar en los campos de la sociología, la geografía, la 
ecología, el urbanismo y diversas perspectivas disciplinares en una especie de reconocimiento de que, al ser un fenómeno complejo, requiere un examen multidisciplinar. En su teoría de la competitividad de las naciones, Porter (1991) plantea que la competitividad es un proceso de generación y transmisión de competencias, que depende de factores macroeconómicos, de las fortalezas que un territorio tiene para crear un adecuado ambiente de negocios que sea generador de riqueza y empleo. Siendo ello posible, solamente cuando se dan las condiciones institucionales, físicas, tecnológicas, sociales, ambientales y de gobernanza.

En este sentido, se reconoce que no sólo las empresas compiten en el mundo globalizado, sino también las regiones y las ciudades. De esta manera la competitividad se ha vinculado con las ciudades por ser estás los principales motores de las actividades económicas, políticas, sociales y de todo tipo. Las ciudades son el espacio primero de realización del capital "marcan en nuestros días, el ritmo de crecimiento, bienestar y progreso, por ello es fundamental entenderlas mejor, analizarlas desde diversos ángulos y generar un repertorio amplio de políticas públicas que nos permitan mejorar la calidad de los espacios urbanos" (Cabrero et al, 2002: 2). Por ser el centro de la vida de las sociedades modernas, espacios en los que habitan más de 3 cuartas partes de la población del planeta, es insoslayable abordar el análisis de las relaciones que guarda la competitividad con otras dimensiones de la vida urbana: la calidad de vida, la generación de oportunidades, la educación, la investigación científica y tecnológica, la configuración urbana de las mismas, el medio ambiente y la sustentabilidad, la riqueza cultura, el papel de las instituciones y la gobernanza, por mencionar algunos.

No son pocos los autores que reconocen la existencia de una relación de doble vía entre competitividad y calidad de vida. Es decir, la competitividad es un medio para incrementar la calidad de vida de las personas y a la vez se convierte en una ventaja competitiva para la atracción de negocios, talentos e incremento en la dinámica económica de una región.

Para autores como Lever y Turok (1992:792) competitividad significa la "producción de bienes y servicios que se enfrentan a la prueba de los mercados a cualquier escala, mientras simultáneamente incrementan su ingreso real, mejoran la calidad de vida de sus habitantes y promueven el desarrollo de una manera sustentable". La calidad de vida se ve reflejada en una mejora constante de las condiciones tangibles e intangibles que definen el bienestar de la gente: infraestructura y servicios de salud, educación, movilidad y recreación; ambiente institucional y de gobernabilidad que propicie la inclusión, la equidad y la participación social en la toma de decisiones, un ambiente natural sustentable, empleo e ingresos dignos, entre otros aspectos.

De acuerdo a Theodore Metaxas (2012:15) en un estudio sobre los determinantes urbanos de la competitividad de las empresas en la región de Thessalonika, Grecia; se manifiestan en las siguientes evidencias:

1. "Las economías de aglomeración están significativamente asociadas con el tamaño del mercado local, la disponibilidad de recursos naturales y la existencia de negocios similares. 
2. "La calidad de vida, relacionada con el trabajo, es tan alta como lo son los valores relacionados con el trabajo disponible, el mayor ambiente cultural y recreativo, y alta calidad de las destrezas locales."

Pero, ¿qué es lo que determina la competitividad?, entendida como la capacidad de una ciudad, país o región para integrarse a los procesos de globalización mejorando a la vez el bienestar y calidad de vida de sus habitantes. Diversos estudiosos han intentado dar respuesta a esta cuestión. Las diferencias tienen su origen en como conciben la competitividad para luego intentar identificar sus determinantes.

Considerando la discusión teórica prevaleciente, es posible observar dos posiciones con relación a los determinantes de la competitividad, cada una de ellas asociada, de alguna manera, con las ideas de Paul Krugman o Michael Porter.

Para el primero el origen de la competitividad está en la propia firma y está relacionado con ganancias en costos y eficiencia que inducen a una mayor productividad. En todo caso los agrupamientos o aglomeraciones entre empresas abonan al mismo propósito generando economías de escala. En otras palabras, el contexto en el que se ubican las empresas no tiene mayor relevancia más allá del elemento geográfico definido por la localización. En tanto, otro importante grupo de estudio se relacionan más con las ideas de M. Porter, para quien el contexto es relevante no sólo por las economías de escala que se pueden desplegar sino también por el conjunto de elementos que inciden en la competitividad y que no están bajo el control de la empresa, tales como el entramado institucional, el papel del gobierno, el medio ambiente, la existencia de complementadores y competidores, entre otros componentes.

Las implicaciones de cada una de las posiciones, en términos de resultados y del papel de los sujetos son distintas:

Para Krugman (PK) y sus seguidores el sujeto, "cuasi único", en la determinación de la competitividad es la empresa, por lotanto, el contexto no es tan relevante, la competitividad es sinónimo de productividad, por lo que es posible esperar consecuencias únicas: ganancias en empleo e ingresos

Para los seguidores de Michael Porter (MP) los sujetos múltiples, uno de ellos es la propia empresa, la competitividad es multideterminada holístico por lo tanto el contexto sí importa, y las consecuencias son diversas: ganancias en empleo, ingresos, mejoras institucionales, mayor inclusión y participación social, mayor responsabilidad ambiental, ye mejoras en el bienestar en general.

Transitamos de una noción en la que la explotación de las ventajas comparativas con base en eficiencia en costos (PK) es suficiente y es el camino cierto para alcanzar la competitividad, a la búsqueda de estrategias diversas en donde el nivel de incertidumbre se incrementa por la complejidad del concepto competitividad y la multiplicidad de sujetos que participan (MP) Igualmente nos movemos de una noción en la que se concibe que ser competitivo equivale a explotar las ventajas comparativas, por tanto, la misma se convierte en un fin en sí mismo y el camino para lograrlo consiste en crear esas ventajas, aunque sea de manera artificial (subvaluación de la moneda, bajos salarios, excepciones fiscales, regulación laxa, etc). A otra 
dónde la competitividad se vincula con el desarrollo y, por tanto, con la calidad de vida y el bienestar de las personas como la opción ineludible para ampliar las oportunidades para el desarrollo de las capacidades de la gente. Y lo que es más importante, la competitividad deja de ser un fin en sí mismo y se convierte en un medio para alcanzar dicho propósito.

\section{La evidencia empírica en la relación entre competitividad y calidad de vida}

Considerando algunos indicadores de impacto en la calidad de vida de los principales municipios turísticos del país, tales como niveles de pobreza, distribución del ingreso, disponibilidad de infraestructura de servicios y desarrollo humano, se observan, en general, aceptables niveles aunque con algunos contrastes, que enseguida comentamos.

Los municipios con mejores indicadores son Benito Juárez, mejor conocido como Cancún en el Estado de Quintana Roo, con un índice de desarrollo humano (IDH) con servicios muy alto (0.906), seguido de Mazatlán Sinaloa, Puerto Vallarta Jalisco y Manzanillo Colima con IDH también muy altos, curiosamente todos son destinos de playa con un modelo turístico tradicional, sustentado en el uso de intensivo de recursos y atractivos naturales.

En contraste, los municipios con los más bajos valores en el IDH no son destinos de playa y a pesar de que presentan niveles de IDH aceptables, mayores a 0.8 (alto), este es menor al indicador observado en los destinos de playa. Entre estos municipios destacan $\mathrm{Sn}$. Miguel de Allende Guanajuato, San Cristóbal de las Casas, Chiapas, e Ixtapan de la Sal en el Estado de México.

Es importante mencionar que los niveles de pobreza son altos, en general, aunque son menores por razones obvias, en aquellos destinos que tienen los IDH mayores.

De los 22 destinos analizados, 9 registran niveles de pobreza mayores al 50\% de su población, y de estos últimos, 3 rebasan el 60\%, San Cristóbal de las Casas Chiapas, San Pedro Mixtepec Oaxaca y San Miguel de Allende Guanajuato. De estos últimos, 2 presentan las disparidades en distribución del ingreso, medidas por el Coeficiente de Gini (CG), más altas; aunque es necesario destacar que el municipio de San Pedro Mixtepec presenta el CG más bajo, lo que significa que el fenómeno de la pobreza está más generalizado entre la población en este municipio.

No hay que olvidar que el CG da cuenta, precisamente, de las disparidades en la distribución del ingreso entre los estratos en que se divide la población, un valor cercano a la unidad indica que el ingreso está casi totalmente concentrado en un estrato; en tanto un valor cercano a cero es indicativo de una distribución más equitativa entre los estratos. 
Tabla 1: Municipios Turísticos de México

\begin{tabular}{|c|c|c|c|c|c|c|c|}
\hline Estado & Ciudad & Población & $\begin{array}{c}\text { Viviendas } \\
\text { particulares } \\
\text { habitadas } \\
\text { que no } \\
\text { disponen de } \\
\text { drenaje } \\
\end{array}$ & Pobreza & $\begin{array}{c}\text { Coeficiente } \\
\text { de Gini }\end{array}$ & $\begin{array}{l}\text { Índice de } \\
\text { drenaje }\end{array}$ & $\begin{array}{c}\text { Índice de } \\
\text { desarrollo } \\
\text { humano } \\
\text { con } \\
\text { servicios }\end{array}$ \\
\hline $\begin{array}{l}\text { Baja } \\
\text { California } \\
\text { Sur }\end{array}$ & La Paz & 283378 & $3.52 \%$ & 24.67439 & 0.4402902 & 0.965 & 0.889 \\
\hline $\begin{array}{c}\text { Baja } \\
\text { California } \\
\text { Sur }\end{array}$ & Los Cabos & 215657 & $4.10 \%$ & 28.53371 & 0.4613635 & 0.966 & 0.878 \\
\hline Colima & Manzanillo & 169058 & $1.78 \%$ & 31.7254 & 0.458032 & 0.985 & 0.892 \\
\hline Chiapas & $\begin{array}{c}\text { San Cristóbal de } \\
\text { las Casas }\end{array}$ & 149924 & $9.40 \%$ & 66.11376 & 0.4976625 & 0.895 & 0.832 \\
\hline Guanajuato & $\begin{array}{l}\text { San Miguel de } \\
\text { Allende }\end{array}$ & 139634 & $23.45 \%$ & 63.68685 & 0.501969 & 0.752 & 0.83 \\
\hline Guanajuato & Guanajuato & 187929 & $7.66 \%$ & 38.31416 & 0.4827872 & 0.903 & 0.868 \\
\hline Guerrero & $\begin{array}{l}\text { Acapulco de } \\
\text { Juárez }\end{array}$ & 785594 & $9.35 \%$ & 51.61682 & 0.4437187 & 0.902 & 0.845 \\
\hline Guerrero & $\begin{array}{l}\text { Zihuatanejo de } \\
\text { Azueta }\end{array}$ & 119945 & $6.04 \%$ & 53.61042 & 0.4081492 & 0.238 & 0.75 \\
\hline Jalisco & Cihuatlán & 25862 & $2.57 \%$ & 55.76595 & 0.3896742 & 0.976 & 0.876 \\
\hline Jalisco & Chapala & 30890 & $1.99 \%$ & 48.08063 & 0.3917832 & 0.983 & 0.883 \\
\hline Jalisco & Puerto Vallarta & 235587 & $1.95 \%$ & 45.5202 & 0.4183812 & 0.981 & 0.892 \\
\hline $\begin{array}{l}\text { Edo. } \\
\text { México }\end{array}$ & Ixtapan de la Sal & 29157 & $11.44 \%$ & 59.54551 & 0.4403098 & 0.885 & 0.83 \\
\hline $\begin{array}{l}\text { Edo. } \\
\text { México }\end{array}$ & Valle de Bravo & 56425 & $9.43 \%$ & 58.28571 & 0.4360403 & 0.903 & 0.853 \\
\hline Morelos & Jojutla & 66658 & $3.04 \%$ & 41.698 & 0.421507 & 0.97 & 0.875 \\
\hline Oaxaca & $\begin{array}{c}\text { San Pedro } \\
\text { Mixtepec -Dto. } \\
22-\end{array}$ & 39657 & $7.56 \%$ & 66.19519 & 0.3907992 & 0.926 & 0.857 \\
\hline Querétaro & Tequisquiapan & 56487 & $6.29 \%$ & 55.39212 & 0.415387 & 0.94 & 0.883 \\
\hline $\begin{array}{l}\text { Quintana } \\
\text { Roo }\end{array}$ & Cozumel & 77421 & $1.81 \%$ & 31.72536 & 0.4164145 & 0.984 & 0.889 \\
\hline $\begin{array}{l}\text { Quintana } \\
\text { Roo }\end{array}$ & Benito Juárez & 327643 & $4.23 \%$ & 8.74531 & 0.450005 & 0.988 & 0.906 \\
\hline $\begin{array}{l}\text { Quintana } \\
\text { Roo }\end{array}$ & Solidaridad & 157161 & $4.42 \%$ & 30.33099 & 0.4440691 & 0.967 & 0.886 \\
\hline Sinaloa & Mazatlán & 426243 & $3.51 \%$ & 28.13548 & 0.4245809 & 0.967 & 0.894 \\
\hline
\end{tabular}




\begin{tabular}{|l|l|l|l|l|l|l|l|}
\hline Yucatán & Mérida & 897331 & $5.78 \%$ & 29.40243 & 0.4494214 & 0.943 & 0.887 \\
\hline
\end{tabular}

\section{Fuente: CONEVAL y SIMBAD}

Para complementar y fortalecer el análisis anterior se realizaron algunos ejercicios estadísticos para determinar si al menos existe asociación (correlación) entre el nivel de competitividad de una muestra de destinos turísticos, con respecto a algunos indicadores de bienestar que inciden en la calidad de vida de la población; pobreza, pobreza extrema, dinámica económica, medioambiente e índice de desarrollo humano con servicios (IDHs).

En una primera correlación, se contrastaron los niveles de pobreza con respecto al resultado obtenido en la dimensión económica del índice de competitividad económica de zonas urbanas (ZU) que elabora el Centro de Investigación y Docencia Económica (CIDE), de una muestra de ellas con una importante dinámica en el turismo, tales como Acapulco de Juárez, Puerto Vallarta, Guanajuato, Manzanillo, Mérida, Mazatlán, La paz, Los Cabos y el municipio de Benito Juárez (Cancún).

Los resultados indican de manera contundente que, al menos, existe una fuerte correlación entre la dinámica económica y la pobreza, a mayor fortaleza económica menor pobreza. No estamos hablando de relaciones de causalidad, pero si es posible concluir que existe una relación inversa en el movimiento de ambas variables; en la medida en que se mejora en la dimensión económica la pobreza se reduce.

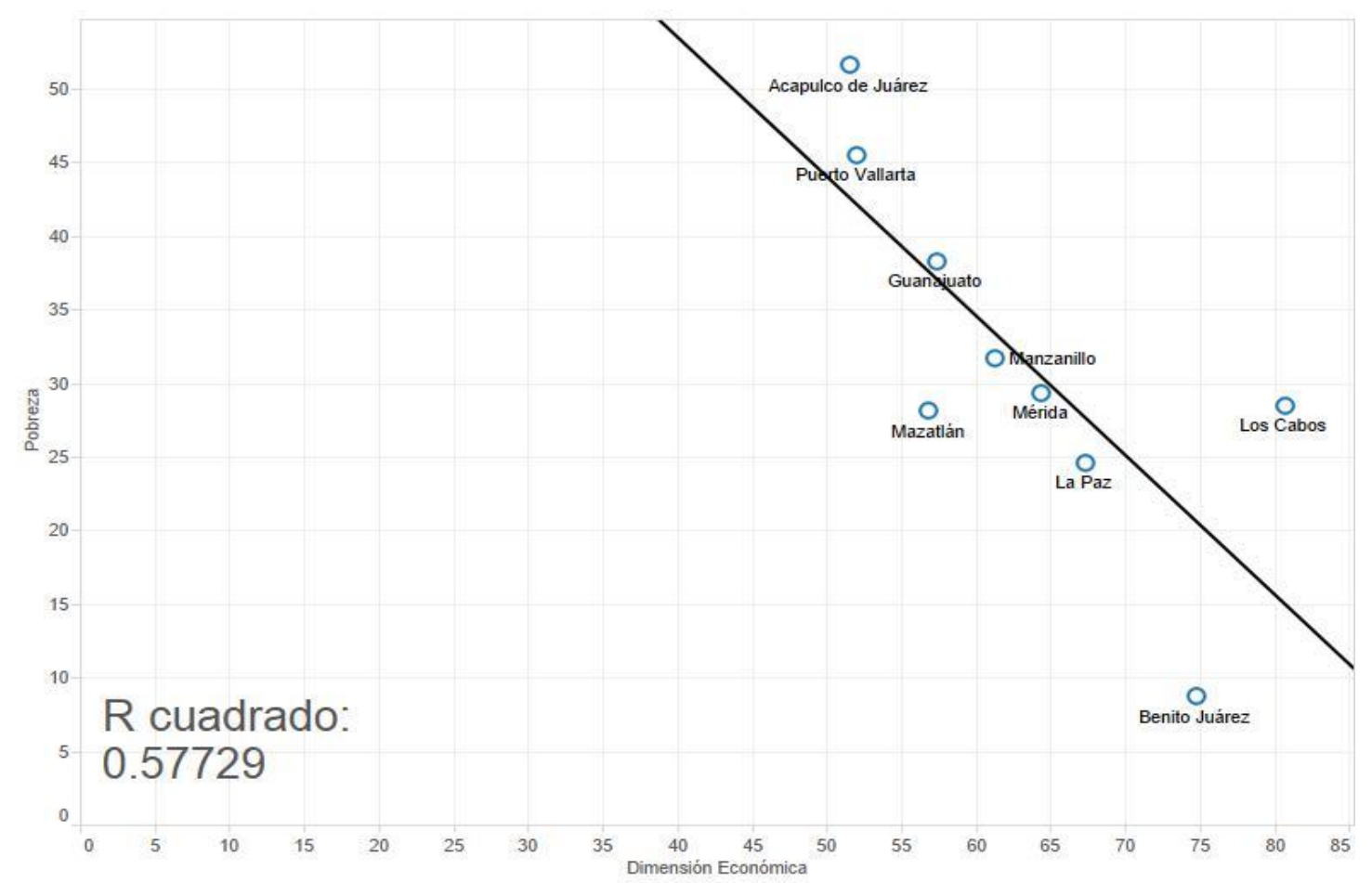

Gráfico 2: Dimensión económica versus pobreza. Fuente: Elaboración propia con base en IMCO, CONEVAL y SIMBAD 
Si bien, la correlación entre la mayor competitividad en la dimensión económica versus el nivel de pobreza es muy alta y permite sacar conclusiones relativamente robustas. No ocurre lo mismo cuando se observan los resultados en las relaciones entre otras variables. En la gráfica siguiente, en donde se representa la correlación entre la dimensión ambiental y la pobreza, está no es tan contundente, aunque si se observa cierto nivel de correspondencia. Mejoras en la dimensión ambiental se correlacionan positivamente con menores niveles de pobreza. Sin embargo se debe insistir en el bajo valor del coeficiente que mide dicha relación.

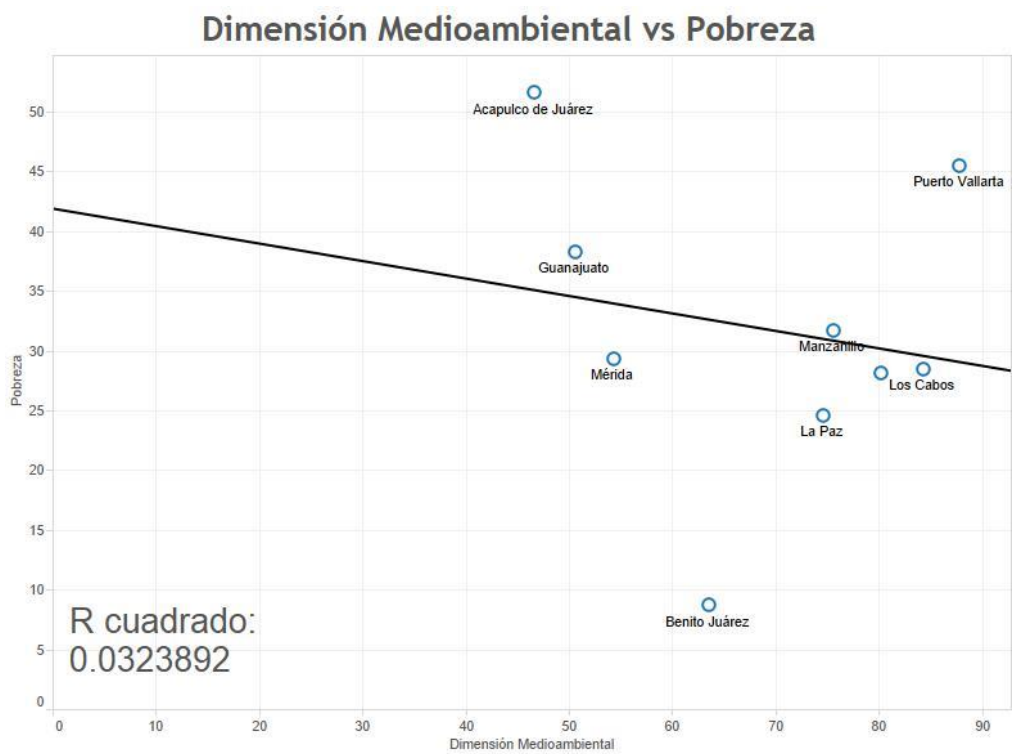

Gráfico 3: Dimensión medioambiental versus pobreza. Fuente: Elaboración propia con base en IMCO, CONEVAL y SIMBAD. Fuente: Elaboración propia con base en IMCO, CONEVAL y SIMBAD

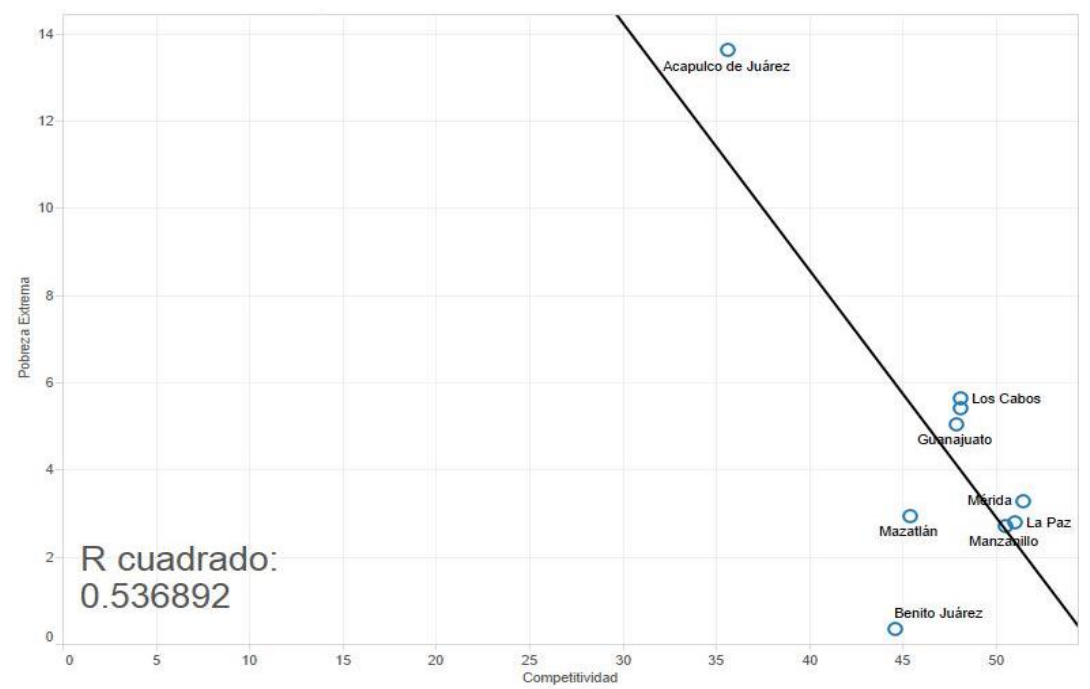

Gráfico 4: Competetividad versus pobreza. Fuente: Elaboración propia con base en IMCO, CONEVAL y SIMBAD. Fuente: Elaboración propia con base en IMCO, CONEVAL y SIMBAD 
No ocurre lo mismo cuando relacionamos Competitividad con pobreza extrema. Al parecer los resultados son contundentes al mostrar claramente una relación inversa en los movimientos de ambas variables; una mayor competitividad se corresponde con menores niveles de pobreza extrema.Igual correlación se observa entre competitividad y pobreza en sentido amplio, aunque con una menor robustez evidenciada por un menor valor en el coeficiente de correlación.

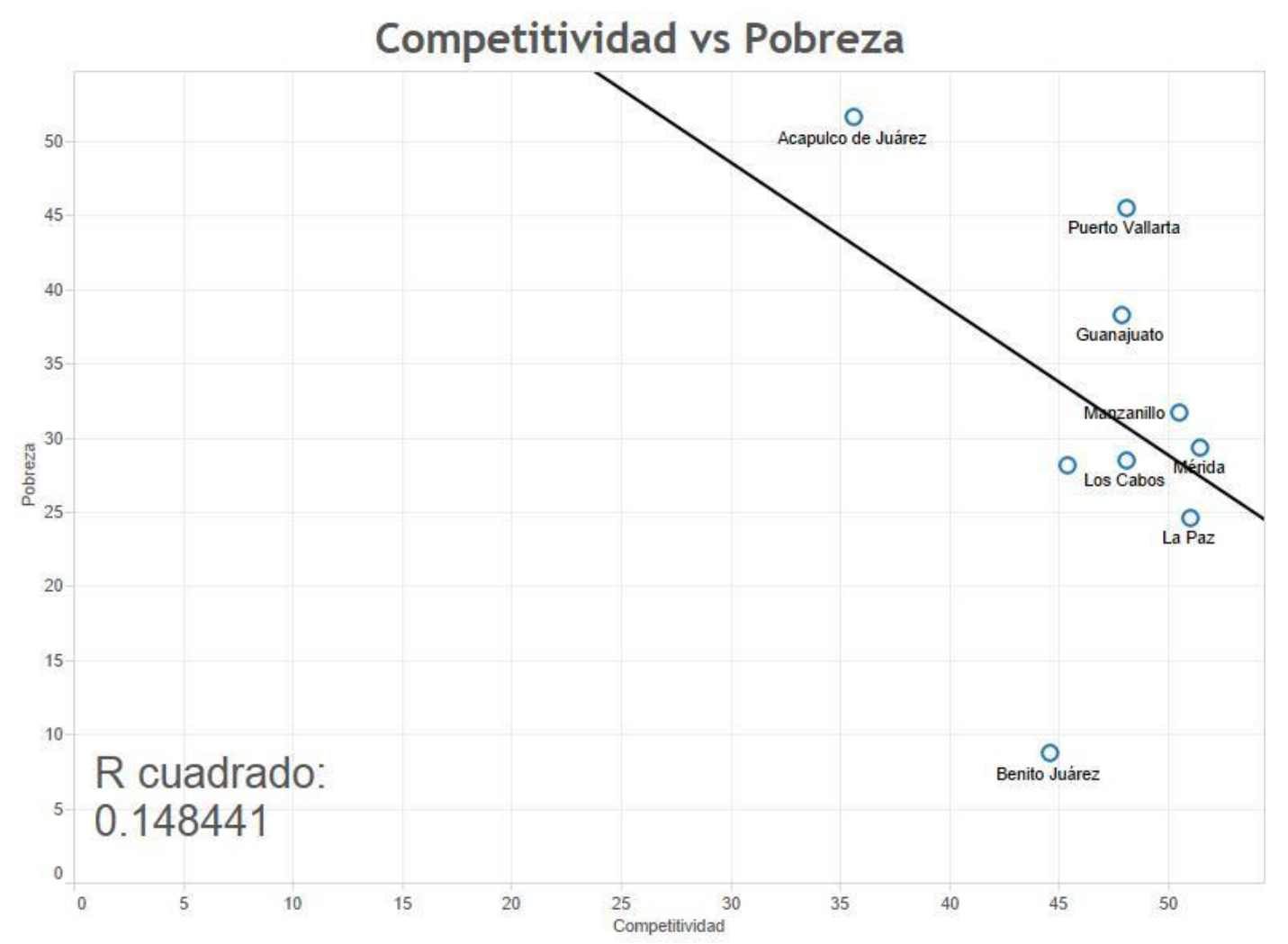

Gráfico 5: Competetividad versus pobreza. Fuente: Elaboración propia con base en IMCO, CONEVAL y SIMBAD. Fuente: Elaboración propia con base en IMCO, CONEVAL y SIMBAD

De igual manera se observa una correlación positiva y directa relativamente importante entre competitividad e índice de desarrollo humano con servicios (IDHs); a mayor competitividad corresponde un IDHs también mayor. Es decir, ambos indicadores se mueven en el mismo sentido. 


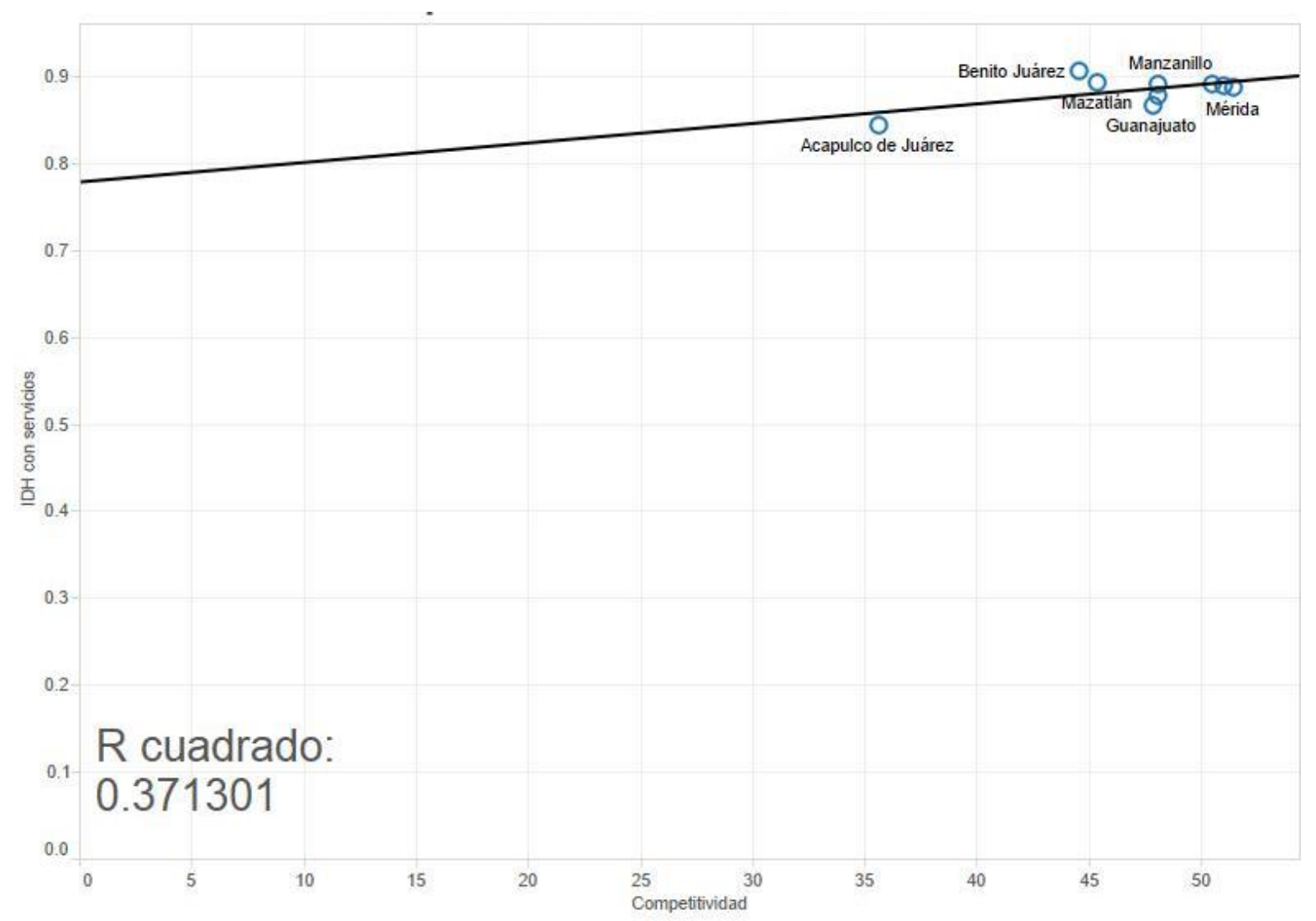

Gráfico 6: Competitividad versus IDH servicios. Fuente: Elaboración propia con base en IMCO, CONEVAL y SIMBAD. Fuente: Elaboración propia con base en IMCO, CONEVAL y SIMBAD

En general se puede aceptar una asociación relativamente importante entre algunas variables que miden la competitividad, la fortaleza económica y las condiciones ambientales, con respecto a variables clave relacionadas con la calidad de vida, tales como la pobreza y el IDHs.

\section{Competitividad, desarrollo humano y bienestar en los principales destinos turísticos de playa}

Considerando únicamente los principales destinos turísticos de playa, Acapulco, Cancún, Los Cabos, Mazatlán y Puerto Vallarta, que tienen una fuerte dinámica económica sustentada en la explotación de sus atractivos naturales se observan resultados interesantes en la relación entre competitividad, medida por el índice de competitividad de ciudades (AU) de México y el IDH que elaboró el Programa de las Naciones Unidad para el Desarrollo (PNUD) para el año 2010.

Lo primero que destaca es la relación relativa que existe entre, competitividad medida por medio del (ICCM), y desarrollo humano, medida por el IDH. El destino con mejor posición en competitividad en el año 2011 es Los Cabos, ocupando el lugar 7 de una muestra de áreas 
urbanas o ciudades. Sin embargo, no es el que registra el más alto IDH, el mismo lo tiene Mazatlán con un IDH de 0.8344 (alto) a pesar de ser el destino peor ubicado en competitividad con el lugar 59, de los cinco destinos aquí analizados.

Acapulco es el pero ubicado en el IDH con un valor de 0.7898 y es el segundo peor ubicado en competitividad, ocupando el lugar 57. Es importante destacar que 3 destinos mantienen una tendencia consistente de mejora en competitividad (IP) del año 2003 al año 2011. Los Cabos en primer lugar, seguido de Cancún y Puerto Vallarta. Salvo en la dimensión urbana, Los Cabos tiene los mayores puntajes en las otras tres dimensiones consideradas por el CIDE en la evaluación de la competitividad; la económica (Eco), la socio demográfica (SD) y la Institucional en donde obtuvo una calificación perfecta (100) en el año 2011.

Tabla 2: Indicadores de competitividad y bienestar

\begin{tabular}{|l|l|l|l|l|l|l|l|l|l|}
\hline Ciudad & $\begin{array}{l}\text { IP } \\
03\end{array}$ & $\begin{array}{l}\text { IP } \\
07\end{array}$ & Eco. & Inst. & SD & Urb. & IP 11 & $\begin{array}{l}\text { ICCM } \\
11\end{array}$ & IDH \\
\hline Acapulco & 50 & 43 & 31 & 48 & 39 & 34 & 38 & 57 & 0.7898 \\
\hline Cancún & 10 & 28 & 46 & 65 & 92 & 39 & 65 & 21 & 0.8292 \\
\hline $\begin{array}{l}\text { Los } \\
\text { Cabos }\end{array}$ & 15 & 34 & 48 & 100 & 92 & 57 & 74 & 7 & 0.8259 \\
\hline Mazatlán & 31 & 26 & 46 & 51 & 78 & 61 & 59 & 59 & 0.8344 \\
\hline $\begin{array}{l}\text { Pto. } \\
\text { Vallarta }\end{array}$ & 22 & 45 & 37 & 75 & 85 & 61 & 65 & 19 & 0.8011 \\
\hline
\end{tabular}

Fuente: CONEVAL, INEGI y PNUD

Si existiera una fuerte relación entre competitividad y calidad de vida, serían los destinos turísticos de Los Cabos, Puerto Vallarta, Cancún, Acapulco y Mazatlán los que debieran registrar los indicadores más altos de bienestar y desarrollo humano. En principio la relación entre competitividad (ICCM) e IDH no es tan contundente, en general.

Tomando en cuenta un set de indicadores de bienestar específicos, se observan los siguientes resultados:

a) El indicador más bajo de mortalidad lo tiene Los Cabos (19.67), seguido de Cancún, Mazatlán, Acapulco y Puerto Vallarta, en ese orden.

b) El índice más alto de cobertura de salud, lo tiene Cancún (0.8565) seguido de Mazatlán, Los Cabos, Acapulco y Puerto Vallarta.

c) En alfabetización (Alfabet.) Los Cabos y Mazatlán ocupan el primer lugar con 96.06 de su población alfabetizada, muy cerca de ellos se ubican Puerto Vallarta y Cancún con más de $95 \%$ de alfabetización, estando a la zaga Acapulco con un porcentaje de $89.53 \%$, nivel alto si consideramos que la media nacional rebaza el $95 \%$.

d) El Ingreso personal promedio anual (Ing. Prom. PC) en dólares americanos es de 12600 para Los Cabos, 12339 para Cancún y 11241 para Mazatlán ocupando 
respectivamente los 3 primeros lugares en ese rubro. Acapulco nuevamente presenta el mayor rezago con 6999 y junto con Puerto Vallarta con 8777 dólares se encuentran por debajo de la media nacional que ronda para el año 2010 los 9700 dólares.

e) En participación política (Ind. ParPol) Mazatlán ocupa el primer lugar con un índice de 0.9777, seguido de Cancún con 0.8966 y Los Cabos con 0.7141, el último lugar es ocupado nuevamente por Acapulco con un valor de 0.4292 en dicho indicador, muy cerca de Puerto Vallarta con 0.4905 .

f) Es importante mencionar que los valores registrados en todos los casos, se reducen si se considera la perspectiva de género. Esto significa que tanto en el acceso a los servicios de salud, de educación, empleo e ingresos que son, por cierto, fundamentales en la explicación de la calidad de vida y el bienestar de las personas, sigue habiendo discriminación de la mujer.

Tabla 3: Indicadores de Desarrollo Humano (Coneval 2013)

\begin{tabular}{|l|l|l|l|l|l|}
\hline & Acapulco & Cancún & Los Cabos & Mazatlán & Pto. Vallarta \\
\hline Mortalidad & 20.90 & 19.67 & 0.2031 & 20.28 & 21.17 \\
\hline Ind. Salud & 0.8460 & 0.8565 & 0.8511 & 0.8514 & 0.8464 \\
\hline Con-Gen. & 0.8311 & 0.8412 & 0.8346 & 0.8364 & 0.8277 \\
\hline Alfabet. & 89.56 & 95.75 & 96.06 & 96.06 & 95.78 \\
\hline Ind. Educ. & 0.8146 & 0.8273 & 0.8194 & 0.8638 & 0.8429 \\
\hline -Género & 0.8143 & 0.8271 & 0.8193 & 0.8638 & 0.8429 \\
\hline Ing. Prom PC & 6996 & 12339 & 12600 & 11241 & 8777 \\
\hline (dls) & & 0.8037 & 0.8072 & 0.7882 & 0.7468 \\
\hline Ind. Ingreso & 0.7090 & 0.7744 & 0.7766 & 0.7580 & 0.7224 \\
\hline -Gen. & 0.6802 & 0.8966 & 0.7141 & 0.9777 & 0.4905 \\
\hline Ind. ParPol. & 0.4256 & 0.8989 & 0.9221 & 0.9158 & 0.9092 \\
\hline IndEmplGen & 0.8893 & 0.8292 & 0.8259 & 0.8344 & 0.8011 \\
\hline IDH & 0.7898 & 1.964 & & \\
\hline
\end{tabular}

Fuente: Consejo Nacional de Evaluación de la Política Social, con base en ENIGH (2010) y Censo Poblacional 2010.

Tabla 4: Condiciones de Pobreza (porcentajes)

\begin{tabular}{|l|l|l|l|l|l|l|l|}
\hline Ciudad & Pobreza & Pob. Ext. & P. Mod. & $\begin{array}{l}\text { No Pob. } \\
\text { No Vuln. }\end{array}$ & $\begin{array}{l}\text { Al menos } \\
\text { 1 carencia }\end{array}$ & $\begin{array}{l}\text { Ingreso } \\
\text { inf. A } \\
\text { líea } \\
\text { Bienestar }\end{array}$ & $\begin{array}{l}\text { Pob } \\
\text { ext/no } \\
\text { pob. No } \\
\text { vuln. }\end{array}$ \\
\hline Acapulco & 51.66 & 13.62 & 37.99 & 13.84 & 81.96 & 55.80 & 0.12 \\
\hline Cancún & 8.74 & 0.36 & 8.38 & 51.12 & 45.66 & 11.95 & 0.07 \\
\hline Los Cabos & 28.53 & 5.63 & 22.89 & 28.58 & 68.82 & 31.13 & 0.11 \\
\hline Mazatlán & 28.13 & 2.95 & 25.20 & 28.32 & 61.07 & 38.74 & 0.13 \\
\hline $\begin{array}{l}\text { Pto. } \\
\text { Vallarta }\end{array}$ & 45.52 & 5.43 & 40.08 & 19.84 & 69.64 & 56.03 & 0.14 \\
\hline
\end{tabular}


Fuente: Consejo Nacional de Evaluación de la Política Social, con base en ENIGH (2010) y Censo Poblacional del 2010.

En cuanto a los indicadores de pobreza, la tendencia se mantiene. Los destinos con los mejores indicadores siguen siendo Cancún y Los Cabos en tanto llama la atención los altos indicadores de pobreza que registran Acapulco y Puerto Vallarta. En lo específico se observa los siguientes resultados:

a) El más alto nivel de pobreza en general lo tiene Acapulco con $51.66 \%$ de su población, seguido por Puerto Vallarta con $45.52 \%$. El mejor posicionado es Cancún con un muy bajo nivel de apenas $8.74 \%$ de su población.

b) Situación similar se guarda en el indicador de pobreza extrema, aunque en este rubro Mazatlán ocupa el segundo lugar con tan sólo $2.95 \%$ de su población ene esa condición.

c) En pobreza moderada el mayor rezago se presenta en Puerto Vallarta con un $40.08 \%$ de su población en esa condición, le sigue Acapulco con $37.99 \%$, siendo Cancún el destino con el mejor indicador con tal sólo $8.38 \%$ de su población en esa condición.

d) Cancún destaca nuevamente por el número no pobres y no vulnerables con el $51.12 \%$ de su población en esa condición. Acapulco tiene el valor más bajo con tan sólo el $13.84 \%$ de su población.

e) En general los indicadores que reflejan las condiciones de pobreza se mueven en el mismo sentido, en general Acapulco registra los mayores rezagos pero en algunos indicadores es Puerto Vallarta el peor posicionado, como ocurre en el indicador de Población con ingreso inferior a la línea de bienestar con una población de $56.06 \%$ contra 55.80\% de Acapulco. El mejor posicionado, con un indicador muy por encima del resto de destinos es Cancún con tan sólo $11.95 \%$ de su población en esta condición.

f) Relacionando la pobreza extrema con los no pobres y no vulnerables el valor más bajo lo tiene Cancún con un coeficiente de 0.07 , lo que quiere decir que por cada 100personas no pobres y no vulnerables, existen 7 personas en pobreza extrema.

\section{Conclusiones}

La competitividad debe ser concebida como una tarea social al tener implicaciones importantes en la calidad de vida y el bienestar de las personas. No es solamente una tarea de las empresas o de los gobiernos en particular pues debido a su complejidad y multidimensionalidad son muchos los jugadores (sujetos) que la hacen posible.

Las empresas son un actor importante, en acuerdo con Paul Krugman, pero no único. De igual manera los elementos contextuales de mercado, medioambientales, sectoriales, de 
gobernanza y de políticas públicas son importantes en su determinación; por lo tanto, el contexto sí importa, tal y como lo reconoce M. Porter.

De igual manera es importante reconocer que debe ser evaluada desde una perspectiva intra e intergeneracional; es decir, sustentable. La competitividad sólo tiene sentido en la medida en que contribuye a la ampliación de las oportunidades de participación social y mejora de la calidad de vida y, por tanto, al abatimiento de la pobreza y la exclusión en cualquiera de sus formas.

Al abordar el análisis de los destinos turísticos de México y en particular los principales destinos de playa que destacan por su importante actividad económica con base en la explotación de sus atractivos naturales, culturales y/o histórico arquitectónicos, se constata cierta relación positiva entre competitividad y calidad de vida, medida a través de algunos indicadores como pobreza, desarrollo humano y otros indicadores específicos como acceso a salud, educación, ingresos, participación política y empleo.

Los casos de Cancún y Los Cabos sobresalen por sus resultados en bienestar, en tanto municipios como Puerto Vallarta y Mazatlán presentan algunos rezagos, mientras que Acapulco se ubica a la zaga en casi todos los indicadores, siendo también el más rezagado en competitividad.

Pareciera haber ya algunas señales de alarma con relación a los altos costos ambientales, sociales y económicos que ponen en tela de juicio la viabilidad de tradicionales destinos turísticos como Acapulco, Puerto Vallarta y Mazatlán como una manifestación, también, del agotamiento del modelo turístico dominante basado en la explotación de la riqueza natural, cultural e histórica de dichos destinos.

Las alternativas no son tan claras, pero parecieran dirigirse hacia modelos que generen una mayor inclusión, un mayor bienestar para las comunidades y que sean sustentables. La compleja realidad a que nos enfrentamos nos obliga a pensar de forma creativa y diferente en el diseño de alternativas de desarrollo para una gran cantidad de comunidades de México que viven del turismo.

\section{Referencias bibliográficas}

Boddy, Martin. (1999). Geographical Economics and Urban Competitiveness: A Critique, Urban Studies, vol. 36, pp. 811-842.

Bubb, Leslie and Amer Hirmis (2004). Conceptual Framework for Regional Competitiveness. Regional Studies, 38:9, 1015-1028. En http//www.tandfonline.com/loi/cres20 Revisado el 12/07/2012

Cabrero Mendoza, Enrique, Orihuela, Isela y Ziccardi, Alicia. Competitividad de las ciudades mexicanas 2007. La nueva agenda de los municipios urbanos. México: 
Secretaría de Economía-CIDE, 2007. [En línea, disponible en http://www.cide.edu/programas/Documento_INDICE_COMPETITIVIDAD_CIUD ADES_Mex_2007.pdf.

Cabrero Mendoza, Enrique, Orihuela, Isela y Ziccardi, Alicia (2002). Competitividad urbana en México: una propuesta de medición. Eure, vol. XXXV-109, pp 79:99, Pontificia Universidad Católica de Chile: Chile.

Cabrero Mendoza, Enrique(Ed)(2013). Retos de la competitividad urbana. CIDE, México, D.F.

Instituto Nacional de Estadística y Geografía (2011). Censos Económicos 2009.

Instituto Nacional de Estadística y Geografía (2011). Censo General de Población y Vivienda 2010

Krugman, Paul R., (1996). Making sense of the competitiveness debate. Oxford Review of Economic Policy, vol.12-3.

Lengyel, Imre (2004). The pyramid-model: Enhaging regional competitiveness in Hungary. Acta Oeconomica No. 3. En www2.eco.uszeged.hu/regions_gazdfejl_szcs/pdf/the_pyramid-model.pdf (consulta 23/08/2012).

Leva Germán, "Indicadores de calidad de vida urbana: Teoría y metodología” Universidad Nacional de Quilmes, Buenos Aires, Argentina, 2005.

Lever, W. y Turok, I. (1999) "Competitive Cities: Introduction to the Review", Revista Urban Studies, $36(5 / 6)$.

Porter, Michael (1990), “The Competitive Advantage of Nations”, Harvard Business Review, Vol. 68, No. 2, pp. 73-93.

Porter, M. (1995) “The competitive advantage of the inner city". Harvard Business Review, $73(3), 55-71$.

Porter, M. (1996) "Competitive Advantage, Agglomeration Economics, and Regional Policy”. International Regional Science Review, 19, (1- 2), 85-93.

Raco, M. (1999). Competition, collaboration and the new industrial districts: examining the institutional turn in local economic development. Urban Studies, vol. 36 (5-6), 951968. 
Rogerson, R. (1999). Quality of life and city competitiveness. Urban Studies, vol.36 (5-6), 969-985.

Salas Zapata, W. Alfredo; Ríos Osorio, L. Alberto; Álvarez del Castillo, Javier(2011). La ciencia emergente de la sustentabilidad: de la práctica científica hacia la constitución de una ciencia. Rev. Interciencia, Vol.36, No.9, Asociación interciencia, Caracas Venezuela.

Secretaria de Turismo-Colegio de México (2011). Análisis económico del mercado laboral en el sector turístico. Hacía una política pública para la igualdad entre hombres y mujeres. En: www.sectur.gob.mx (20/08/2013)

Sandoval Cabrera, Pablo. Importancia de la contabilidad y fiscalidad ambiental: situación actual y alternativas de implementación en el subsector turismo de México. Versión Mimeografiada.

Siggel, Eckhard. (2007). International Competitiveness and Comparative Advantage: A Survey and a Proposal for Measurement. CESinfo, Venice Summer Institute.

Sobrino, Jaime, "Competitividad territorial: ámbitos e indicadores de análisis. En: Economía, Sociedad y Territorio, Dossier especial”, 2004.

Turok, Ivan (2010). Cities, regions and Competitiviness. Regional Studies, 38:9, 1069-1083. En http://dx.doi.org/10.1080/0034340042000292647. Consulta 12/08/2012.

Yap, Josef (2004). A Note on the Competitiveness Debate, 1:20, Discussion paper series 2004-39. Philippine Institute for Development Studies. En: The Economic and Social Research Council: www.esrc.ac.vk

www.sectur.gob.mx

www.datatur.sectur.gob.mx 